\title{
Transcriptomic Biomarker Pathways Associated with Death in HIV-infected Patients with Cryptococcal Meningitis
}

Irina St. Louis ( $\square$ irinast|@umn.edu )

University of Minnesota Twin Cities https://orcid.org/0000-0003-0795-4723

Abdu K Musubire

Makerere University School of Medicine

David B Meya

Makerere University School of Medicine

Henry W Nabeta

Makerere University School of Medicine

Hesham Mohei

University of Minnesota

David R Boulware

University of Minnesota

Paul R Bohjanen

University of Minnesota

\section{Research article}

Keywords: fatal immune reconstitution inflammatory syndrome, transcriptomic biomarkers, AIDS/HIV, cryptococcal meningitis IRIS

Posted Date: September 21st, 2020

DOI: https://doi.org/10.21203/rs.3.rs-50764/v1

License: (a) (1) This work is licensed under a Creative Commons Attribution 4.0 International License. Read Full License

Version of Record: A version of this preprint was published at BMC Medical Genomics on April 16th, 2021. See the published version at https://doi.org/10.1186/s12920-021-00914-1. 


\section{Abstract}

Background: Cryptococcal meningitis (CM) is a major cause of death in HIV-infected patients in subSaharan Africa. Many CM patients experience cryptococcosis-associated immune reconstitution inflammatory syndrome (C-IRIS), which is often fatal. We sought to identify transcriptomic biomarker pathways in peripheral blood that are associated with, or, predict the development of death or fatal C-IRIS among patients with CM who were enrolled in the Cryptococcal Optimal ART Timing (COAT) Trial.

Methods: We assessed peripheral blood gene expression using next-generation RNA sequencing in 4 groups of patients with CM: 1) no C-IRIS or Death; 2) C-IRIS survivors; 3) fatal C-IRIS; 4) Death without CIRIS. Gene expression was assessed at the time of ART initiation, at 1, 4, and 8 weeks on ART, and at the time of C-IRIS events.

Results: We identified 12 inflammatory and stress response pathways, including interferon type 1 signaling, that were upregulated at the time of ART initiation in patients with future fatal C-IRIS, as compared with survivors. The upregulation of transcripts involved in innate immunity (inflammasome, Toll-like receptor signaling), was observed at the time of fatal or nonfatal C-IRIS events. At the time of fatal C-IRIS events, numerous transcripts within fMLP, Rho family GTPases, HMGB1, and other acute phase response signaling pathways were upregulated, which reflect the severity of inflammation and systemic oxidative stress. Those who died without recognized C-IRIS also had increased expression of pathways associated with oxidative stress and tissue damage. The Partial Least Squares model estimated above mentioned pathways as predictors of fatal outcome.

Conclusions: Our results showed that overactivated innate immunity, involving Toll-like receptor/inflammasome, and inflammation-induced oxidative stress, are associated with fatal outcomes. The results of this study provide insight into the molecular drivers of death and fatal C-IRIS to inform future diagnostic test development or guide targeted treatments.

\section{Background}

Cryptococcal meningitis (CM) is the most prevalent fungal opportunistic infection in persons living with AIDS, with CD $4+T$ cell counts of less than 100 cells/uL. HIV-associated cryptococcal infections account for one-fifth of the million deaths annually, in Sub-Saharan Africa $[1,2]$. The pathogenesis of death in CM is largely unknown. Many CM patients die due to a disseminated fungal infection that includes the involvement of the brain, and improved antifungal treatment regimens are much needed $[3,4]$. Yet even with effective antifungal therapy, up to $20 \%$ of cryptococcal patients with CM die after initiating antiretroviral therapy (ART) due to cryptococcal immune reconstitution inflammatory syndrome (C-IRIS), an exaggerated and dysregulated inflammatory response that as occurs as the immune system begins recovery [5]. C-IRIS often presents as clinical worsening in the setting of effective antifungal and antiviral therapy [6]. In this study, we evaluated transcriptome of peripheral blood of patients treated for CM who 
survived or died after ART initiation, in order to identify biomarker pathways associated with death and better understand the molecular pathogenesis of fatal C-IRIS.

The samples used for this study were collected from patients with CM during the Cryptococcal Optimal ART Timing (COAT) Trial [7]. This was a randomized clinical trial to assess survival in cryptococcal patients who received earlier ART, initiated within 1-2 weeks of antifungal therapy (early arm), or after approximately 4-6 weeks of antifungal therapy (deferred arm). The trial concluded that deferring ART for 4-6 weeks had a $15 \%$ improved survival [7]. The incidence of C-IRIS, did not differ significantly between

the earlier-ART arm and the deferred-ART arm. However, mortality from C-IRIS appeared to be higher when ART was initiated earlier [7, 8].

In this study, we performed longitudinal transcriptomic analyses using next-generation RNA sequencing on whole blood samples from patients with CM in the COAT Trial who survived or died and compared them with patients who did not experienced C-IRIS or die. We observed that fatal C-IRIS was associated with the upregulation of interferon type 1 and STAT pathways before ART initiation. At the time of C-IRIS, upregulation of transcripts involved in innate immunity (inflammasome and toll-like receptor signaling) was observed in C-IRIS survivors, and many of these same transcripts were upregulated to even higher levels in those who died from C-IRIS. Patients who died but did not have C-IRIS had increased expression of transcripts encoding components of pro-inflammatory pathways associated with oxidative stress and tissue damage. These same pathways were also upregulated at the time of fatal C-IRIS events. These data demonstrated that patients with $\mathrm{CM}$ who died or developed fatal C-IRIS had distinct transcriptomic signatures that may have diagnostic and prognostic values.

\section{Methods}

\section{Sample collection and RNA isolation}

This study used deidentified pre-existing samples obtained from the COAT trial performed in Kampala, Uganda. COAT trial was approved by University of Minnesota IRB (\# 0810M49622), including the approval and consents for samples collection and storage for genetic testing). For more information, and Ethics and Consent to Participate, visit https://clinicaltrials.gov/ct2/show/NCT01075152. During the trial period, $115 \mathrm{HIV}$-infected subjects in Kampala with confirmed CM were randomized to early versus deferred ART at approximately 1 week post-diagnosis and then followed for 26 weeks [7]. Study protocol, ethics approval for the multi-site parent study, and the criteria for C-IRIS diagnosis have previously been published [7]. Deidentified samples processing and analysis had been performed at the University of Minnesota (IRB exempt ID STUDY00005386). For the current study, whole blood (2.5 mL) was collected into PAXgene tubes (QIAGEN Inc.) from 68 subjects was assessed by next generation RNA sequencing. Whole blood samples were collected at the time of ART initiation (week 0), at weeks 1, 4 and 8 after ART commencement and at time of C-IRIS events (Figure 1). We categorized patients into 2 groups based on their randomization in the COAT trial: earlier ART initiation arm and deferred ART initiation arm. Patients in each arm were then placed into groups based on their clinical course and outcomes: 1) No C-IRIS or 
Death Group; 2) C-IRIS Survivor Group; 3) Fatal C-IRIS Group; and 4) Death without C-IRIS Group . Cases of death or C-IRIS occurred within the first month of the ART (see Figure 1).

\section{Next generation RNA sequencing}

Total ribonucleic acid (RNA) was extracted from whole blood using PAXgene Blood RNA kits (QIAGEN Inc.) according to the manufacturer's protocol, and quantity was measured by RiboGreen RNA Quantification. One microgram of RNA was submitted to University of Minnesota Biomedical Genomics facility for quality controls, assessed by Agilent 2100Bioanalyzer (Agilent Technologies). The Clontech StrandedRNA Pico Mammalian kit was used for library creation.

Paired-end (2x125bp) sequencing was done on HiSeq2500 instrument, for 125 cycles, v4 chemistry (Illumina). The fastq files from this study are deposited in the National Center for Biotechnology Information's Gene Expression Omnibus and are accessible through GEO Series ticket number no. .

\section{Bioinformatical analysis of gene expression}

Fastq files were processed through the bioinformatic pipeline Minnesota Supercomputing institute Unix interface: the qualities of raw reads were assessed and trimmed (in Trimmomatic), if necessary. The alignment and mapping of sequencing reads to a reference transcriptome (hg38) was performed with HISAT2 (hierarchical indexing for spliced alignment of transcripts). The normalization to FPKM (Fragments Per Kilobase of transcript per Million mapped reads) and quantification the expression of the transcripts was performed with Cuffquant (Cufflinks workflow).

The identification of transcripts that were differentially expressed were done in JMP14pro and Partek. The Principal component analysis (PCA) visualized specimen-specific gene expression as PC correlations between groups (survived vs. died) and subgroups (see figure 1). The list of differentially expressed genes was obtained from analysis of variance with restricted maximum likelihood (ANOVA-REML). Significantly changed probe intensities were filtered based on a minimum fold change threshold of 2 for up- or downregulation. Values of $P$ were corrected for multiple testing using the Benjamini-Hochberg false discovery rate.

The enrichment of functional gene categories/pathways was performed in Ingenuity pathway analysis (IPA) statistical software. IPA's pathway refers to a group of functionally or structurally related genes (with known function) that jointly form a network. The IPA workflow comprised core, functional, and canonical pathway analyses and was used as a reference data set. Both direct and indirect molecular relationships were included in the analysis settings, and the significance of relationships between 2000 immune gene transcripts was indicated with z-score and Fisher's exact test $P$ values $<.05$, with false Benjamini-Hochberg discovery rate (FDR) correction. 


\section{Biomarkers Search}

For C-IRIS and Death biomarker identification, the nonlinear iterative partial least squares (PLSs) algorithm with leave- one-out cross-validation method (available in JMP14 Pro (SAS Institute Inc.) was used, which was carried out on 2000 immune transcripts. Variable Importance in the Projection (VIP) scores were extracted to reveal relations between predictors and outcome and plotted against coefficient of regression. Predictors and responses were scaled to have a mean of 0 and a standard deviation of 1 (by dividing each column by its standard deviation). To consider the transcript as a potential biomarker, the filters were set as such: the VIP score was $>0.8$ and the regression correlation coefficient was $>0.2$ in at least 1 of the studied subgroups. Model coefficients with their respective ranked VIP contributions are presented in Supplemental table 3. The algorithm is available upon request.

\section{Results}

\section{Gene expression patterns differed among patients who developed C-IRIS, survived or died}

Our goal was to identify gene expression signatures in peripheral blood associated with the development of death or fatal C-IRIS among patients with CM. We performed next generation RNA sequencing of samples from 68 HIV-infected subjects with CM who participated in the COAT Trial. Of these, 39 subjects had been randomized to initiate ART within two weeks (earlier arm) and 29 subjects had been randomized to initiate ART after 5 weeks (deferred arm) [7]. All subjects received induction anti-fungal therapy with Amphotericin B and fluconazole, followed by consolidation and maintenance therapy with fluconazole as described previously [7]. Of the 68 subjects, 10 subjects in each arm did not develop C-IRIS or death (Control Group)), 10 subjects in each arm had C-IRIS and survived (C-IRIS Survivor Group), and 9 subjects in each arm died without C-IRIS (Death without C-IRIS Group). Ten subjects from the earlier arm had C-IRIS and died (Fatal C-IRIS Group; see Figure 1). Cases of C-IRIS included those classified based on predefined criteria in [6]. All cases of death or C-IRIS occurred within the first month of the ART treatment. Peripheral blood was collected before ART initiation (week 0), at weeks 1, 4 and 8 on ART, and at the time of C-IRIS (Figure 1). Groups had no significant differences in age, pre-ART HIV viral load, or CD4+ T cell count (Supplemental figure 1). The serum cryptococcal antigen titers, as measured by LFA (lateral flow immunoassay), were significantly higher in the C-IRIS Survivor Group, as compared to patients who died in the earlier study arm with or without C-IRIS (Supplemental figure 1).

We hypothesized that patients with $\mathrm{CM}$ would show distinct gene expression signatures in the blood that could distinguish between the four groups: 1) No C-IRIS or Death (control), 2) C-IRIS Survivor Group), 3) Fatal C-IRIS Group, and 4) Death without C-IRIS Group. We applied a principal component analysis (PCA), to visualize the differences in gene expression levels in the entire dataset (26398 expressed RNAs for each sample) (Figure 2A). Principal component analysis was used to separate gene expression in 211 individual samples from the 68 subjects. The results separated the samples into four clusters: samples 
from patients who survived (excluding samples collected at the time of C-IRIS events, yellow dots), samples from those who died (excluding samples collected at the time of C-IRIS events, blue dots), and samples collected at C-IRIS events (red, died; green, survived). As shown in figure 2A, samples collected from the C-IRIS Survivor Group at the time of C-IRIS events clustered separately from samples collected from the Fatal C-IRIS Group at the time of fatal C-IRIS events.

We performed additional PCA analysis in order to cluster samples based on expression of significant immune transcripts among samples collected at week 0 (pre-ART), from all patient groups (Figure 2B). The list of all expressed transcripts was truncated to 2200 immune genes, and the top 3 principal components were extracted. The immune gene expression at week 0 showed distinct separation comparing patients who later died of C-IRIS from those who survived (shown as PCA\#1, black, grey and green dots, in figure 2B). These data show that gene expression in those who died could be readily distinguished from those who survived already at the time of ART initiation (week 0 ), suggesting that gene expression signatures could potentially be used as prognostic markers to define risk for death. These results prompted us to perform further data mining to reveal biological information in gene sets that may contribute to fatal events.

\section{At week 0 transcriptome profiles differed between surviving patients who did or did not develop C-IRIS}

Transcript expression was further compared between groups by ANOVA-REML, using criteria described in the methods. Comparing gene expression at week 0 in surviving patients who did not develop C-IRIS (Control Group) to those who developed C-IRIS (C-IRIS Survivor Group), C-IRIS survivors exhibited lower baseline expression of transcripts associated with antiviral responses, including interferon-induced protein family members, interferon-induced proteins with tetratricopeptide repeats, and oligoadenylate synthetases (IFIs, IFITs, ISGs, and OASs respectively) (Supplemental figure 2). This confirmed our previously published observation that lower expression of genes encoding interferon type 1-inducible transcripts at the time of CM diagnosis are associated with subsequent nonfatal C-IRIS events [9]. Additionally, we identified elevated expression of transcripts encoding markers of complement components (C1QA, C1QB, C1QC, CFD), HLA-DRB (1 and 5), and IL12 pathways in the C-IRIS Survivor Group at week 0 , in comparison to the Control Group ( $p<0.05$, no FDR correction, see Supplemental table 1). The complement components, HLA-DRs and IL12 play important roles in antigen presentation, which suggests that prior to ART commencement, monocytes and antigen-presenting cells are already activated in C-IRIS survivors. These results suggest that we can use gene expression biomarkers to identify patients at risk for the development of C-IRIS prior to their initiation of ART [9].

\section{At week 0 transcriptome profiles differed between patients who survived or died}


We compared gene expression at week 0 in ten patients from the earlier ART arm who subsequently died from C-IRIS (Fatal C-IRIS Group) to week 0 control patients (No C-IRIS or Death Group) in both study arms. Interestingly, the patients who died from C-IRIS did not exhibit significant deficiency in the expression of interferon-response pathway genes, as compared to controls. These results were different from the result described above where patients with C-IRIS who survived had lower expression of these genes. Using Ingenuity Pathway Analysis (IPA) software we combined transcript expression measurements over pathways to identify which pathways act as markers in predicting or explaining fatal outcomes. We ran a comparison analysis of canonical these pathways and built the heatmaps of the top up- and downregulated pathways.

We identified twelve inflammatory immune pathways that were upregulated at week 0 in the Fatal C-IRIS Group as compared to the three other groups from either arm of study: No C-IRIS or Death Group (Control), C-IRIS Survivor Group, and Death without C-IRIS Group. Since the results were almost identical, we combined groups from both study arms together (see Figure 3A, columns 1, 2, 3, respectively). These 12 pathways included cytokines and other pro-inflammatory molecules such as CXCL1, CXCR1, ICAM1, IL6, IL8, IL11, interferons, and components of MAPK signaling (MAPK14, NFKBIA, IL1 receptors). No significantly downregulated pathways were identified, suggesting that patients who died due to C-IRIS, were not immunocompromised to a higher degree than patients from other groups based on lack of immune gene expression. Notably, patients from Death without C-IRIS Group did not show any statistically significant upregulation of immune gene expression, except for type 1 interferon signaling, when compared to the No C-IRIS or Death (Control), or the C-IRIS Survivor Group (week 0). The Death without C-IRIS Group, however, exhibited a trend towards a downregulated expression of various HLA-, Th1- and Th2- pathway encoded genes, but upregulation of PD1/PDL1 at the time of ART initiation, which may reflect the adaptive immune cell exhaustion ( $z$ scores $<1$ ). The fold change in expression values for these transcripts are presented in the Supplemental table 2. Together, these results suggest that gene expression at week 0 differed between the Fatal C-IRIS Group, the Death without C-IRIS group and those of survivors (Figure $3 \mathrm{~A}$ ).

\section{Changes in gene expression caused by ART initiation, in earlier and deferred ART initiation arms has differed among those who died and survived}

Using same pathway-based biomarker discovery approach, we assessed changes in pathway expression over time among patients in the earlier and deferred ART initiation arms. At one week of observation on ART, studied groups exhibit upregulation of similar components of pro-inflammatory pathways, when compared to week 0 within each corresponding arm (Figure 3B). Patients in the Death without C-IRIS Group, whether they were in the earlier or deferred ART arms, showed a distinct shift toward upregulation of transcripts involved in oxidative phosphorylation, high mobility group box 1 proteins (HMGB1), proinflammatory cytokine signaling (IL6, 8, 15), and Rho/GTPase pathways (Figure 3B, columns 1, 2). A 
similar trend was seen in Fatal C-IRIS Group at one week after ART initiation (Figure 3B, column 3). Interestingly, Ras homologous Guanosine diphosphate (GDP)-dissociation inhibitors, RhoGDIs, which negatively regulate Rho family GTPases, were downregulated in all Death Groups (with or without C-IRIS), at 1 week after ART initiation. Thus, No C-IRIS or Death Groups (with or without C-IRIS), showed distinct gene expression changes, yet, independently from the timing of ART initiation.

Control groups (in earlier and deferred arms) did not exhibit significant changes at week 1 , in the above mentioned pathways, or other proinflammatory pathways, as compared to corresponding week 0 (Figure 3B, column 4, 5). Patients in the C-IRIS Survivor Group from the earlier ART arm exhibited more dramatic upregulation of proinflammatory gene expression as compared to those in the C-IRIS Survivor Group who were in the deferred ART arm (Figure 3B, column 6, 7).

Comparison between samples, collected at week one post-ART showed that the Death without C-IRIS Group exhibited the most pronounced changes in gene expression in earlier and deferred ART arms (Figure $3 \mathrm{C}$ ). Numerous transcripts within granulocyte- activation pathways, such as $\mathrm{N}$-formyl-Met-Leu-Phe (fMLP pathway), HMGB1, and Rho family GTPases (Rho GTPases), were upregulated in the Death without C-IRIS Group, as compared to the controls. The oxidative phosphorylation pathway was the foremost overexpressed, followed by Rho family GTPases and stress kinases (Figure 3C, column 1). The Fatal C-IRIS Group exhibited similar gene expression changes, when compared to controls (Figure 3C, column 2); however, when compared to Death without C-IRIS Groups (earlier and deferred arms combined), the oxidative stress and neutrophil involvement signatures were not present at week 1 of observation (Figure 3C, column 3). In comparison, C-IRIS survivors have shown significantly lower expression of 10 out of 12 pathways presented in figure 3 (see column 4) when compared to Death without C-IRIS Groups. These results indicate that the transcriptomic signature of Death without C-IRIS Group in the blood samples collected at the closest time point to death is different than in those of comparators.

\section{Immune recovery over 8 weeks on ART in control patients without C-IRIS or death}

Longitudinal analysis of immune reconstitution during 8 weeks on ART in the No C-IRIS or Death (control) Groups, who had favorable clinical recovery, revealed downregulation of interferon signaling and NFkappa B signaling pathways, but upregulation of phagocyte maturation pathways (Figure 3D, columns 1 , 2). This course of immune recovery on ART was similar to what we previously demonstrated in advanced stage HIV-infected patients without opportunistic infections after initiation of ART [10]. We also assessed changes in gene expression in the C-IRIS Survivor Group in the period following C-IRIS events. The C-IRIS Survivors Group exhibited downregulation of eight signaling pathways at week 8 on ART (most proximal post-C-IRIS time point) when compared to the gene expression at C-IRIS events (Figure 3D, columns 3, 4). For example, Toll-like receptor signaling, pro-inflammatory IL6, IL8, and T cell exhaustion pathways were downregulated, which may represent a sign of recovery from chronic antigen exposure and a prolonged 
stage of chronic inflammation [11]. Additionally, transcripts involved in Th1 and Th2 pathways showed a trend towards upregulation, but this trend did not pass the threshold of a significant z-score (Figure 3D, columns 3 , 4). Perhaps, assessment of longer time points of evaluation, such as 12 and 26 weeks postART, would show a more significant recovery in expression of T cell pathways.

\section{Dysregulated immune gene expression at the time of fatal C-IRIS}

The major goal of this study was to identify altered gene expression at fatal C-IRIS events in order to better understand the molecular pathology of fatal C-IRIS. Analysis of changed gene expression at the time of fatal C-IRIS revealed many significantly upregulated acute phase response and oxidative stress pathways (e.g. IL1 and IL6, TLR, HMGB1, NRF2-mediated, NFkB, p38-MAPK), when compared to the most proximal longitudinal time point (W1) within the fatal C-IRIS group (Figure 3E column 1), or the No C-IRIS or Death (control) Group at week 1 (Figure 3E column 2).

Since fatal C-IRIS cases occurred within the 4 weeks of ART initiation and only in the earlier ART arm, we compared gene expression in the Fatal C-IRIS Group to the Control Group collected at week 4 post-ART in the earlier ART arm (Figure 3E column 3). Fatal C-IRIS showed significant upregulation of 14 inflammatory pathways, implying that during favorable immune reconstitution, effective antifungal treatment and ART, the acute inflammation accompanied by reactive oxygen species production are downregulated within the first month of treatment.

Transcripts involved in acute phase response pathways, Toll-like receptor signaling, IL1,6, p38 MAPK were upregulated in the fatal C-IRIS group as compared to C-IRIS Survivor Groups, during C-IRIS events (Figure 3E, column 4). The lower expression of transcripts involved in Th1, CD28 and other T cell- related pathways, but upregulation of transcripts involved in T cell exhaustion signaling pathways (PD1/PDL1), was observed during fatal C-IRIS events when compared to C-IRIS events in the C-IRIS Survivor Group (Figure 3E, column 4).

In comparison to Death without C-IRIS group, fatal C-IRIS showed the upregulation of the same acute phase response pathways, a similar expression of HMGB1 and T cell exhaustion signaling, but downregulation of complement and oxidative stress pathways (eNOS, NRF2-mediated, Rho family GTPases, fMLP, etc.) (Figure 3E, column 5). These results indicate a divergence between activation pathways that are associated with death without C-IRIS and death due to C-IRIS.

\section{Transcriptomic biomarkers predict fatal C-IRIS or death}

To identify transcripts that may be predictive biomarkers of fatal C-IRIS events, we used a probability modeling based on a partial least squares (PLS) computational algorithm, as described in the methods. PLS analysis was performed for samples from the C-IRIS Survivor Group and Fatal C-IRIS Group, with 
comparisons to the rest of the groups, including all time points within each group. The models were carried out on the list of 2200 molecules that are significantly enriched in immune pathways.

The model revealed that most of the top-ranked biomarkers were distinct and specific for either fatal or non-fatal C-IRIS events, and the rest were similar between these groups. For example, differential expression of transcripts encoding IL15, IL31, integrins (ITGA7, ITGB2), and SIGLECs (sialic acid binding immunoglobulin - like lectins) were ranked as highest importance for nonfatal C-IRIS events (C-IRIS Survivor Groups), but not for fatal C-IRIS event (see Table 1). Conversely, p38 MAPK signaling, IL1R, IL18R, TLR1,2,4, NLRP8,12, and transcripts encoding CLECs (C-type lectins), ranked as higher importance for fatal C-IRIS (Fatal C-IRIS Group), but not for C-IRIS survivors (Figure 4). 
Table 1

The comparison of transcriptomic biomarkers between fatal and nonfatal C-IRIS.

\begin{tabular}{|c|c|c|c|c|c|c|}
\hline \multirow{2}{*}{$\begin{array}{l}\text { Gene } \\
\text { Symbol }\end{array}$} & \multicolumn{2}{|c|}{ Fatal C-IRIS Group } & \multicolumn{2}{|c|}{$\begin{array}{l}\text { C-IRIS Survivor Group, earlier } \\
\text { arm }\end{array}$} & \multicolumn{2}{|c|}{$\begin{array}{l}\text { C-IRIS Survivor Group, deferred } \\
\text { arm }\end{array}$} \\
\hline & $\begin{array}{l}\text { R for } \\
\text { VIP }\end{array}$ & VIP rank & $R$ for VIP & VIP rank & $R$ for VIP & VIP rank \\
\hline LIMK1 & -0.0116 & - & 0.0054 & ++++ & 0.015 & ++++ \\
\hline ALOX5 & -0.0101 & - & 0.0026 & ++ & 0.0102 & +++ \\
\hline CTSC & -0.0087 & - & 0.0056 & ++++ & 0.0137 & ++++ \\
\hline IL17REL & -0.0092 & - & 0.0033 & ++ & 0.0074 & ++ \\
\hline TLR10 & -0.0089 & - & 0.0027 & ++ & 0.012 & +++ \\
\hline C3AR1 & -0.0086 & - & 0.0058 & ++++ & 0.012 & +++ \\
\hline CTSD & -0.0064 & - & 0.0038 & +++ & 0.0054 & ++ \\
\hline CTSK & -0.0086 & - & 0.0045 & +++ & 0.0103 & +++ \\
\hline DEFB115 & -0.007 & - & 0.0057 & ++++ & 0.0122 & +++ \\
\hline IL17D & -0.0068 & - & 0.0036 & ++ & 0.0111 & +++ \\
\hline ITGA7 & -0.0077 & - & 0.0058 & ++++ & 0.0086 & ++ \\
\hline ITGB2 & -0.0067 & - & 0.0052 & +++ & 0.0086 & ++ \\
\hline JAG1 & -0.0085 & - & 0.0034 & ++ & 0.011 & +++ \\
\hline SIGLEC10 & -0.0074 & - & 0.0065 & ++++ & 0.0118 & +++ \\
\hline ALPL & -0.0044 & - & 0.0063 & ++++ & 0.0103 & +++ \\
\hline CASP5 & -0.0042 & - & 0.0045 & +++ & 0.0121 & +++ \\
\hline DEFA5 & -0.0044 & - & 0.0047 & +++ & 0.0047 & + \\
\hline ITGAX & -0.0061 & - & 0.0069 & +++++ & 0.0106 & +++ \\
\hline NOD2 & -0.0043 & - & 0.0043 & +++ & 0.0054 & ++ \\
\hline NOTCH2 & -0.0045 & - & 0.0048 & +++ & 0.0063 & ++ \\
\hline NOX1 & -0.004 & - & 0.0048 & +++ & 0.0044 & + \\
\hline SIGLEC5 & -0.0044 & - & 0.0037 & +++ & 0.0084 & ++ \\
\hline SIGLEC7 & -0.0057 & - & 0.0047 & +++ & 0.0091 & +++ \\
\hline BEX1 & -0.0033 & - & 0.0027 & ++ & 0.0026 & + \\
\hline CASP2 & -0.0034 & - & 0.0038 & +++ & 0.0063 & ++ \\
\hline
\end{tabular}




\begin{tabular}{|c|c|c|c|c|c|c|}
\hline ITGA5 & -0.0034 & - & 0.0056 & ++++ & 0.0043 & + \\
\hline SIRPA & -0.0018 & - & 0.0038 & +++ & 0.007 & ++ \\
\hline IL1RAP & 0.0146 & ++++ & -0.002 & - & -0.0071 & - \\
\hline MMP20 & 0.0117 & +++ & -0.0037 & - & -0.0086 & - \\
\hline ALOX5AP & 0.0087 & ++++ & -0.0035 & - & -0.0073 & - \\
\hline CCL1 & 0.0103 & ++++ & -0.004 & - & -0.0079 & - \\
\hline CD55 & 0.0089 & ++++ & -0.0005 & & -0.0041 & - \\
\hline CLEC4D & 0.0102 & ++++ & -0.0027 & - & -0.0055 & - \\
\hline CLEC4E & 0.0106 & ++++ & -0.0049 & - & -0.0068 & - \\
\hline CLEC5A & 0.0091 & ++++ & -0.0032 & - & -0.004 & - \\
\hline F2RL2 & 0.0088 & ++++ & -0.0011 & - & -0.0058 & - \\
\hline FADD & 0.0102 & ++++ & -0.0037 & - & -0.0071 & - \\
\hline FAM65B & 0.0091 & ++++ & -0.0006 & - & -0.0029 & - \\
\hline IFNAR1 & 0.0094 & ++++ & -0.0018 & - & -0.0037 & - \\
\hline IL18R1 & 0.0106 & ++++ & -0.0018 & - & -0.0032 & - \\
\hline IL18RAP & 0.0101 & ++++ & -0.0016 & - & -0.0049 & - \\
\hline IL1R1 & 0.01 & ++++ & -0.0019 & - & -0.0052 & - \\
\hline IRAK3 & 0.0088 & ++++ & -0.0007 & - & -0.0042 & - \\
\hline KLKP1 & 0.0102 & ++++ & -0.0038 & - & -0.0064 & - \\
\hline LILRB5 & 0.0106 & ++++ & -0.0013 & - & -0.006 & - \\
\hline TLR4 & 0.0096 & ++++ & -0.003 & - & -0.0046 & - \\
\hline CASP4 & 0.0065 & +++ & -0.003 & - & -0.0021 & - \\
\hline CD59 & 0.0082 & +++ & -0.0003 & & -0.0003 & \\
\hline CLEC2L & 0.0063 & +++ & -0.0044 & - & -0.0102 & - \\
\hline CXCR1 & 0.0065 & +++ & -0.0011 & - & -0.0004 & \\
\hline EXOSC4 & 0.0081 & +++ & -0.0018 & - & -0.002 & - \\
\hline FAM188A & 0.0076 & +++ & -0.0031 & - & -0.0049 & - \\
\hline IFNGR1 & 0.0066 & +++ & -0.0002 & & -0.0017 & \\
\hline
\end{tabular}




\begin{tabular}{|c|c|c|c|c|c|c|}
\hline IL17F & 0.0086 & +++ & -0.0028 & - & -0.0043 & - \\
\hline IL1R2 & 0.0084 & +++ & -0.0006 & & -0.0054 & - \\
\hline KLF2 & 0.0072 & +++ & 0.0018 & + & -0.0025 & - \\
\hline KLF6 & 0.0064 & +++ & -0.0008 & - & -0.0022 & - \\
\hline MMP8 & 0.0068 & +++ & -0.0006 & & -0.006 & - \\
\hline CLEC4F & 0.0039 & ++ & -0.0036 & - & -0.0065 & - \\
\hline IFRD1 & 0.005 & ++ & 0.0009 & + & -0.0028 & - \\
\hline IL1RL1 & 0.0048 & ++ & -0.0032 & - & -0.0045 & - \\
\hline IL1RN & 0.0049 & ++ & -0.0028 & - & -0.0016 & \\
\hline MMP9 & 0.0057 & ++ & 0.0004 & & -0.0042 & - \\
\hline MOSPD2 & 0.0044 & ++ & 0.0001 & & -0.0019 & - \\
\hline TLR2 & 0.0059 & ++ & -0.0007 & & -0.0006 & \\
\hline HIV_RNA & 0.0017 & + & -0.0018 & - & -0.0015 & \\
\hline IL1RL2 & 0.0032 & + & 0.0023 & ++ & -0.0011 & \\
\hline SIGLEC9 & 0.0023 & + & 0.003 & ++ & 0.0038 & + \\
\hline IFNAR2 & 0.0008 & & 0.0025 & ++ & 0.0019 & + \\
\hline NLRP3 & -0.0009 & - & 0.0054 & ++++ & 0.0079 & ++ \\
\hline \multicolumn{7}{|c|}{$\begin{array}{l}\text { VIP, Variable Importance in the projection values. } \\
\text { R for VIP, Correlation Coefficient of Least Square Mean for VIP. } \\
\text { number of "-" is a visual representation for predictive importance strength that the following } \\
\text { transcript's expression is negatively associated with occurrence of IRIS events. One "-" is equal } 0.7 \text { on } \\
\text { VIP scale. } \\
\text { number of "+" is a visual representation for predictive importance strength that the following } \\
\text { transcript's expression is positively associated with occurrence of IRIS events. One "+" is equal } 0.7 \text { on } \\
\text { VIP scale. }\end{array}$} \\
\hline
\end{tabular}

PLS model identified the list of novel contributive transcripts with high importance for Death without CIRIS Groups (earlier and deferred arms combined), but not for fatal or nonfatal C-IRISs. These included C1QTNF2 and 5, CD207, CD209, CXCL3 and 11, CCL28, MIP, defensins (DEFA6, DEFB107A, DEFB116, DEFB118, etc.) (Supplemental table 3).

Additionally, PLS model identified the high importance of many interferon-response genes, CLEC4F, NLRP5, CD4, CD1C, CCL21, CCR10, as week 0 predictors of subsequent nonfatal C-IRIS. This model also identified FN1 (fibronectin), LILRB4, SERPINE1, CD80, HLA-DQB2, FERM2 (fermitin 2), RND2, CCDC42 
(Rho family GTPases), IL11, IL31, and many other transcripts within several stress response kinases pathways, to be week 0 predictors for subsequent fatal C-IRIS. Conversely, biomarkers such as NLRP6, C3, C5, CTSO, AGTR1, CDH9, etc. were identified as week 0 predictors of Death without C-IRIS Groups.

The complete listing of immune biomarkers with a quantitative estimation of the discriminatory power of each predictor transcript provided by means of VIP scores and the correlation coefficient (R for VIP) is presented in Supplemental Table 3. We were unable to provide pathways comparisons since pathways for many novel top-ranked biomarkers identified by PLS were not available in IPA. Overall, our results suggest that immune and inflammatory biomarkers that we discovered, could be useful for identifying patients with $\mathrm{CM}$ who are at risk for dying from C-IRIS.

\section{Discussion}

Despite improved clinical care in patients with advanced HIV associated CM, overall mortality remains high, highlighting the need for a better molecular understanding of the pathophysiology of fatal outcomes. The biomarkers that we identified in this study provide insight into the pathophysiology of CIRIS and may be useful for identifying patients with $\mathrm{CM}$ who are at the highest risk for death [12]. We identified two gene expression patterns associated with death in patients with $\mathrm{CM}$. The first pattern was seen in patients who developed C-IRIS and died, and the second pattern was seen in patients who died from CM but did not develop C-IRIS.

We have previously reported that HIV-infected patients with CM show a transcriptomic signature of aberrant innate immune activation in the blood during non-fatal C-IRIS events [9]. We showed that C-IRIS survivors exhibited significantly lower expression of transcripts encoding interferon type 2 and antiviral defense proteins prior to ART commencement. Patients who experienced fatal C-IRIS did not show downregulation of interferon-response genes prior to ART initiation, which was one feature that set apart this group from survivors. We suggest that screening of IFN-response gene expression in patients prior to ART initiation, could potentially be used to estimate the risk of deadly C-IRIS development [13]. Additionally, we identified numerous molecules within 12 upregulated proinflammatory pathways (Fig. 3A), prior to ART commencement. Upregulation of these pathways may predispose patients to detrimental clinical sequela after ART initiation, perhaps by promoting the exaggerated inflammatory response associated with innate immune system activation in fatal C-IRIS [14, 15].

The effect of timing of ART initiation in the earlier and deferred arms of the COAT trial did not significantly influence proinflammatory gene expression in the control groups (Fig. 3B). The increase of the oxidative stress response pathway was observed in the C-IRIS survivors from the earlier arm, as compared to the C-IRIS survivors from the deferred arm. ART-induced shift in gene expression in the Fatal C-IRIS Group had more similarity with Death without C-IRIS Groups (from both study arms) than with CIRIS survivors. Activation of pro-inflammatory pathways listed in Figs. 3B and 3C, suggests that individual fungal antigen burden may play a more important role in driving the fatal outcome. This indicates that assessing the effectiveness of antifungal therapy to achieve fungal clearance before 
initiation of ART is important to prevent fatal outcomes. Longitudinal analysis of immune recovery on ART in the groups of survivors was similar to previously demonstrated, when downregulation of innate immune proinflammatory pathways was observed after 8 weeks of observation (Fig. 3D) [10]. The reduced expression of these pathways appears to be beneficial for effective immune reconstitution on ART.

In this study, we identified commonly upregulated pathways, between fatal C-IRIS and C-IRIS survivors, such as TLR- and inflammasome pathways, with the observation that the magnitude of the upregulation of the inflammatory innate immune signature was more drastic in the fatal C-IRIS group. In comparison to patients who survived C-IRIS events, patients who subsequently died from C-IRIS showed a unique immune signature that included upregulation of many acute phase response signaling pathways (e.g. IL1, IL6), and PD1/PDL1 T cell exhaustion signaling pathways (Fig. 3E).

We identified several common molecular pathways between the Fatal C-IRIS and Death without C-IRIS groups, such as fMLP, HMGB1, Rho family GTPases, and stress response kinase pathways (Fig. 3E). Although the upregulation of these pathways in the Fatal C-IRIS Group was not as high compared to the Death without C-IRIS Group, upregulated pathways such as TLR- and inflammasome pathways (which are common with those of C-IRIS survivors) perhaps contributed to fatal outcomes during C-IRIS. Cumulatively, these pathways are part of oxidative stress responses and innate immune defenses often described in relation to neuroinflammation, and very likely reflect the degree of systemic inflammation [16]. FMLP, RhoGDI (Ras homologous GDP dissociation inhibitors) and Rho family GTPases are essential pathways for proper neutrophil activation and function [17]. They involve activation of multi-subunit enzymatic cascades (e.g. NADPH oxidases), which produce reactive oxygen species (ROS) and reactive nitrogen intermediates [18]. Future investigations into the precise role for neutrophils and fMLP pathway, the Rho family GTPases, and their inhibitors RhoGDI during oxidative stress in fatal outcomes may lead to future therapies search that would balance intracellular production of reactive oxygen species [19].

Based on the pathway analyses and predictor screening model results, we composed a map of transcriptional biomarker pathways that appear to drive inflammation during fatal C-IRIS (Fig. 4). This working model included seven predominant immune pathways that are altered during Fatal C-IRIS events, and also in those of survivors and those who died without C-IRIS. These pathways represent an activated innate immune cell signaling in response to both, pathogens and endogenous danger signals, on the background of CD4 + T lymphocytes exhaustion (based on overexpression of PD1/PDL1 pathways, Fig. 3E) [20].

The proposed molecular pathogenesis could be that prior to fatal events, severe and systemic oxidative stress results in cellular necrosis and apoptosis of immune and non-immune cells via pathways depicted in Fig. 4 [21]. Released HMGB1 protein promotes neutrophil recruitment from the bloodstream to brain tissues and back, by means of inflammatory changes of the endothelium and sub-endothelium of the blood-brain barrier [22, 23]. HMGB1 is an endogenous alarmin (danger signal molecule) that mediates the release of chemokines, pro-inflammatory cytokines, and lipid mediators [22, 24]. Reactive oxygen 
intermediates, released by activated granulocytes, set up a damaging environment, accompanied by the loss of endothelial barrier integrity and the trafficking of inflammatory cells across the endothelial barriers [25]. The overproduction of ROS by monocytes and neutrophils in combination with the overactive inflammasome pathway sets up a systemic inflammation that can be fatal to the host.

\section{Conclusions}

Further work into an understanding of the molecular drivers of death and C-IRIS in patients with CM is needed to develop better diagnostic and prognostic tests and guide novel targeted treatments.

\section{Abbreviations}

IRF, Interferon regulatory factor; IFI, interferon alpha inducible protein; IFIT, interferon induced protein with tetratricopeptide repeats; IFNG, interferon gamma, RSAD2, radical S-adenosyl methionine domain containing 2; OAS, 2'-5'-oligoadenylate synthetases; DHX58, DExH-box helicase (LGP2); DDX58, DExD/Hbox helicase 58 (RIG1); C1QB, C1QC, components of complement; TREM1, triggering receptor expressed on myeloid cells 1; Rho GTPases, Ras homologous family GTPases; fMLP pathway, N-formyl-Met-LeuPhe; HMGB1, high mobility group box-1; VIP, variable importance for the projection score; NRF2, nuclear factor erythroid 2 (NF-E2) - related factor; FN1, fibronectin1; C1QTNF2, Complement C1q And Tumor Necrosis Factor-Related; PAMP, pathogen-associated molecular patterns; CD207/CLEC4K, C-type lectin (CTL)/C-type lectin-like (CTLD) domain; CD209/CLEC4L; CLEC4F, CLEC, C-type lectin domain family members; CXCL3 and 11, C-X-C Motif Chemokine Ligands; SIGLEC, sialic acid binding immunoglobulin like lectins; RSAD2, Radical S-Adenosyl Methionine Domain Containing 2; RhoGDI, Ras homologous GDP dissociation inhibitors, GDIs; CCL28, C-C Motif Chemokine Ligand; MIP/CCL3L3, C-C motif chemokine ligand 3 like 3; DEFA6, DEFB107A, DEFB116, DEFB118 defensins A/B; NLRP5,6, NLR (NOD-like receptor) Family Pyrin Domain Containing 5; CCL21, C-C Motif Chemokine Ligand 21; CCR10, C-C Motif Chemokine Receptor 10; LILRB4, Leukocyte Immunoglobulin Like Receptor B4; SERPINE1, Serpin Family E Member 1; FERM2, fermitin 2; RND2, Rho Family GTPase 2; CCDC42 Coiled-Coil Domain Containing 42; C3, C5, complement components; AGTR1, angiotensin II receptor type 1; CDH9, cadherin 9; CTSO, cathepsin 0; NADPHO, Nicotinamide adenine dinucleotide phosphate (NADPH) oxidases; ROS, Reactive Oxygen Species; RNI, reactive nitrogen intermediates.

\section{Declarations}

\section{Acknowledgement}

This work was supported by a UMN departmental start-up fund to I.V-S. and National Institutes of Health grant Al072068 to P.R.B. We acknowledge the patients, clinical and research staff of the parent COAT study. We thank Samar Shahid and Calandra Sagarsky for experimental assistance. We thank Juan E. Abrahante Lloréns for the help with sequence alignment and normalization. 


\section{Authors' contributions}

D.B.M., D.R.B., H.W.N., A.K.M., P.R.B. conducted clinical trial; I.V-S. directed the genomic study and data analysis; H.M. performed experiments; I.V-S., A.K.M., and P.R.B. wrote and edited the manuscript. All authors read and approved the final manuscript.

\section{Competing interests}

All authors: No competed or conflicts of interest reported. All authors have read and approved the manuscript.

\section{Funding}

This work was supported in part by Al096925 (to P.R.B), and department of Medicine start-up fund to I.VS. The funding for parent trial was via U01AI089244 [7]. The funding body did not have any role in study design, data collection and analysis, decision to publish, or preparation of the manuscript.

\section{Availability of data and materials}

The fastq files from this study are deposited in the National Center for Biotechnology Information's Gene Expression Omnibus and are accessible through GEO Series ticket number no. TBD.

\section{Ethics approval and consent to participate}

The approval for the clinical trial, including transcriptomic testing was obtained from the ethics committee of Uganda National Council for Science and Technology, and the institutional review boards in Uganda and at the University of Minnesota (UMN, USA). Written informed consent was obtained from all of the participants or their next of kin in accordance with local institutional review board guidelines of Uganda National Council for Science and Technology. UMN IRB \# 0810 M49622.

\section{Consent for publication}

Not applicable.

\section{Competing interests}

The authors declare that they have no competing interests. 


\section{Author details}

${ }^{1}$ University of Minnesota, Department of Medicine, Minneapolis, MN, USA.

${ }^{2}$ Infectious Disease Institute, Makerere University, Kampala, Uganda.

\section{References}

1. Rajasingham R, Rhein J, Klammer K, Musubire A, Nabeta H, Akampurira A, Mossel EC, Williams DA, Boxrud DJ, Crabtree MB, et al. Epidemiology of meningitis in an HIV-infected Ugandan cohort. Am J Trop Med Hyg. 2015;92(2):274-9.

2. Rajasingham R, Smith RM, Park BJ, Jarvis JN, Govender NP, Chiller TM, Denning DW, Loyse A, Boulware DR. Global burden of disease of HIV-associated cryptococcal meningitis: an updated analysis. Lancet Infect Dis. 2017;17(8):873-81.

3. Tenforde MW, Gertz AM, Lawrence DS, Wills NK, Guthrie BL, Farquhar C, Jarvis JN. Mortality from HIV-associated meningitis in sub-Saharan Africa: a systematic review and meta-analysis. J Int AIDS Soc. 2020;23(1):e25416.

4. Patel RKK, Leeme T, Azzo C, Tlhako N, Tsholo K, Tawanana EO, Molefi M, Mosepele M, Lawrence DS, Mokomane M, et al. High Mortality in HIV-Associated Cryptococcal Meningitis Patients Treated With Amphotericin B-Based Therapy Under Routine Care Conditions in Africa. Open Forum Infect Dis. 2018;5(11):ofy267.

5. Boulware DR, Meya DB, Bergemann TL, Wiesner DL, Rhein J, Musubire A, Lee SJ, Kambugu A, Janoff EN, Bohjanen PR. Clinical features and serum biomarkers in HIV immune reconstitution inflammatory syndrome after cryptococcal meningitis: a prospective cohort study. PLoS Med. 2010;7(12):e1000384.

6. Haddow LJ, Colebunders R, Meintjes G, Lawn SD, Elliott JH, Manabe YC, Bohjanen PR, Sungkanuparph S, Easterbrook PJ, French MA, et al. Cryptococcal immune reconstitution inflammatory syndrome in HIV-1-infected individuals: proposed clinical case definitions. Lancet Infect Dis. 2010;10(11):791-802.

7. Boulware DR, Meya DB, Muzoora C, Rolfes MA, Huppler Hullsiek K, Musubire A, Taseera K, Nabeta HW, Schutz C, Williams DA, et al. Timing of antiretroviral therapy after diagnosis of cryptococcal meningitis. N Engl J Med. 2014;370(26):2487-98.

8. Scriven JE, Rhein J, Hullsiek KH, von Hohenberg M, Linder G, Rolfes MA, Williams DA, Taseera K, Meya DB, Meintjes G, et al. Early ART After Cryptococcal Meningitis Is Associated With Cerebrospinal Fluid Pleocytosis and Macrophage Activation in a Multisite Randomized Trial. J Infect Dis. 2015;212(5):769-78.

9. Vlasova-St Louis I, Chang CC, Shahid S, French MA, Bohjanen PR. Transcriptomic Predictors of Paradoxical Cryptococcosis-Associated Immune Reconstitution Inflammatory Syndrome. Open Forum Infect Dis. 2018;5(7):ofy157. 
10. Boulware DR, Meya DB, Bergemann TL, Williams D, Vlasova-St Louis IA, Rhein J, Staddon J, Kambugu A, Janoff EN, Bohjanen PR. Antiretroviral therapy down-regulates innate antiviral response genes in patients with AIDS in sub-saharan Africa. J Acquir Immune Defic Syndr. 2010;55(4):428-38.

11. Lee I, Huttemann M. Energy crisis: the role of oxidative phosphorylation in acute inflammation and sepsis. Biochim Biophys Acta. 2014;1842(9):1579-86.

12. Chang CC, Dorasamy AA, Gosnell BI, Elliott JH, Spelman T, Omarjee S, Naranbhai V, Coovadia Y, Ndung'u T, Moosa MY, et al. Clinical and mycological predictors of cryptococcosis-associated immune reconstitution inflammatory syndrome. AIDS. 2013;27(13):2089-99.

13. Mohei H, Kellampalli U, Vlasova SL. Immune Reconstitution Disorders: Spotlight on Interferons. International journal of biomedical investigation. 2019;2(1):1-21.

14. Muema DM, Akilimali NA, Ndumnego OC, Rasehlo SS, Durgiah R, Ojwach DBA, Ismail N, Dong M, Moodley A, Dong KL, et al. Association between the cytokine storm, immune cell dynamics, and viral replicative capacity in hyperacute HIV infection. BMC Med. 2020;18(1):81.

15. Dagenais-Lussier X, Loucif H, Murira A, Laulhé X, Stäger S, Lamarre A, van Grevenynghe J. Sustained IFN-I Expression during Established Persistent Viral Infection: A "Bad Seed" for Protective Immunity. Viruses. 2017;10(1):1-11.

16. Wang J, Song Y, Chen Z, Leng SX. Connection between Systemic Inflammation and Neuroinflammation Underlies Neuroprotective Mechanism of Several Phytochemicals in Neurodegenerative Diseases. Oxid Med Cell Longev. 2018;2018:1972714.

17. Mócsai A, Walzog B, Lowell CA. Intracellular signalling during neutrophil recruitment. Cardiovasc Res. 2015;107(3):373-85.

18. Zeng MY, Miralda I, Armstrong CL, Uriarte SM, Bagaitkar J. The roles of NADPH oxidase in modulating neutrophil effector responses. Mol Oral Microbiol. 2019;34(2):27-38.

19. Haider L. Inflammation, Iron, Energy Failure, and Oxidative Stress in the Pathogenesis of Multiple Sclerosis. Oxid Med Cell Longev. 2015;2015:725370.

20. Newton K, Dixit VM. Signaling in innate immunity and inflammation. Cold Spring Harb Perspect Biol. 2012;4(3):1-19.

21. Westman J, Grinstein S, Marques PE. Phagocytosis of Necrotic Debris at Sites of Injury and Inflammation. Front Immunol. 2019;10:3030.

22. Frank MG, Weber MD, Watkins LR, Maier SF. Stress sounds the alarmin: The role of the dangerassociated molecular pattern HMGB1 in stress-induced neuroinflammatory priming. Brain Behav Immun. 2015;48:1-7.

23. Nguyen GT, Green ER, Mecsas J. Neutrophils to the ROScue: Mechanisms of NADPH Oxidase Activation and Bacterial Resistance. Front Cell Infect Microbiol. 2017;7:373.

24. Kany S, Vollrath JT, Relja B. Cytokines in Inflammatory Disease. Int J Mol Sci 2019, 20(23).

25. Mittal M, Siddiqui MR, Tran K, Reddy SP, Malik AB. Reactive oxygen species in inflammation and tissue injury. Antioxid Redox Signal. 2014;20(7):1126-67. 


\section{Figures}

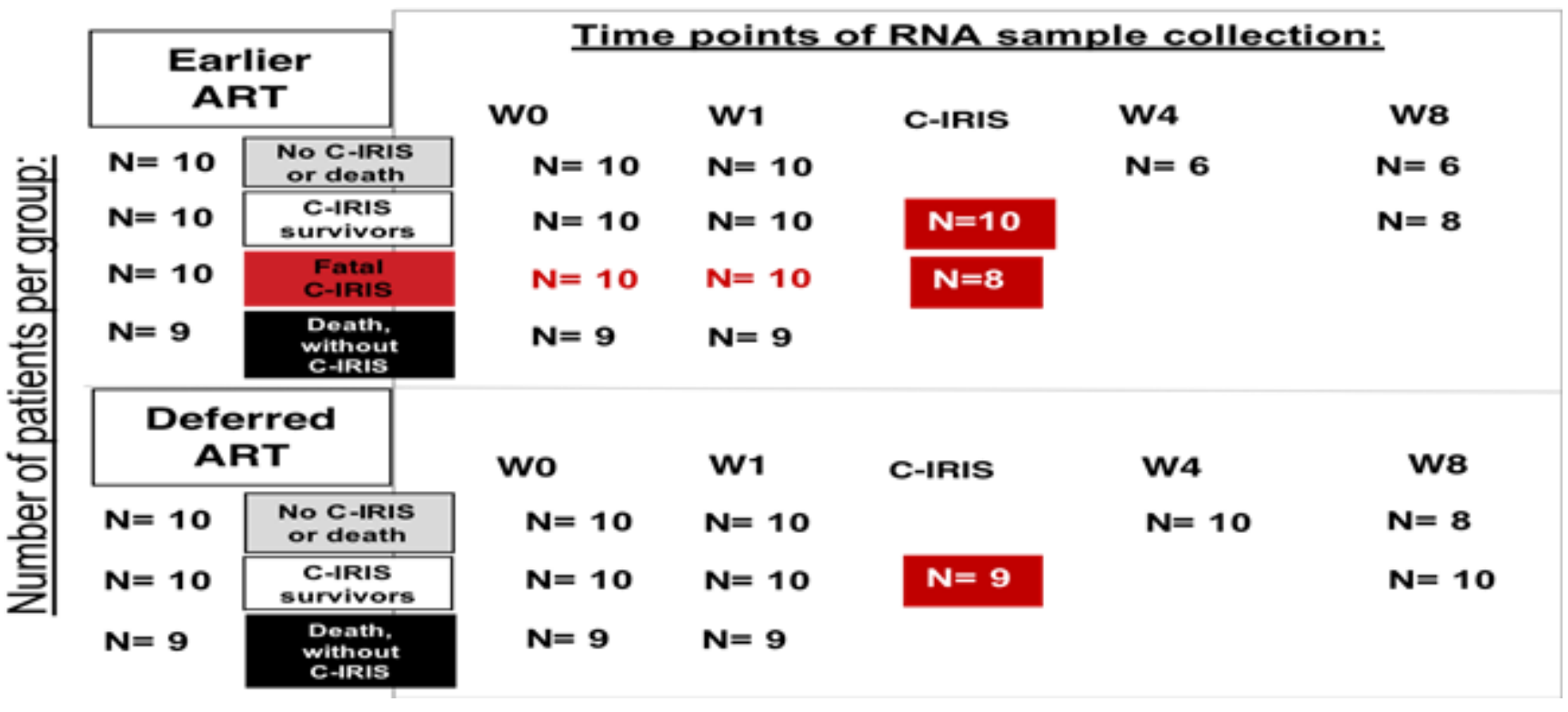

Figure 1

Sample collection and timeline. Blood samples were collected prospectively from HIV-infected Ugandans with CM who were enrolled in the COAT Trial. After COAT study completion, samples from 68 patients were chosen for transcriptome analyses. Of these patients, 39 had been randomized to the earlier ART arm of the COAT Trial, and 29 had been randomized to the deferred ART arm. Ten subjects from each arm did not develop C-IRIS or death (No C-IRIS or Death), 10 subjects in each arm had C-IRIS and survived (CIRIS Survivors), and 9 subjects in each arm died without C-IRIS (Death without C-IRIS). Ten subjects from the earlier arm had C-IRIS and died (Fatal C-IRIS). At the far left of the figure, the number of patients in each of these groups is shown. Blood was collected at randomization immediately prior to ART initiation (W0), one week after ART initiation (W1), at week 4 (W4) and week 8 (W8) after ART initiation, and at the time of C-IRIS events (C-IRIS). The number of samples collected and analyzed at each of these time points for each group of patients is shown. 


\section{$2 A$.}

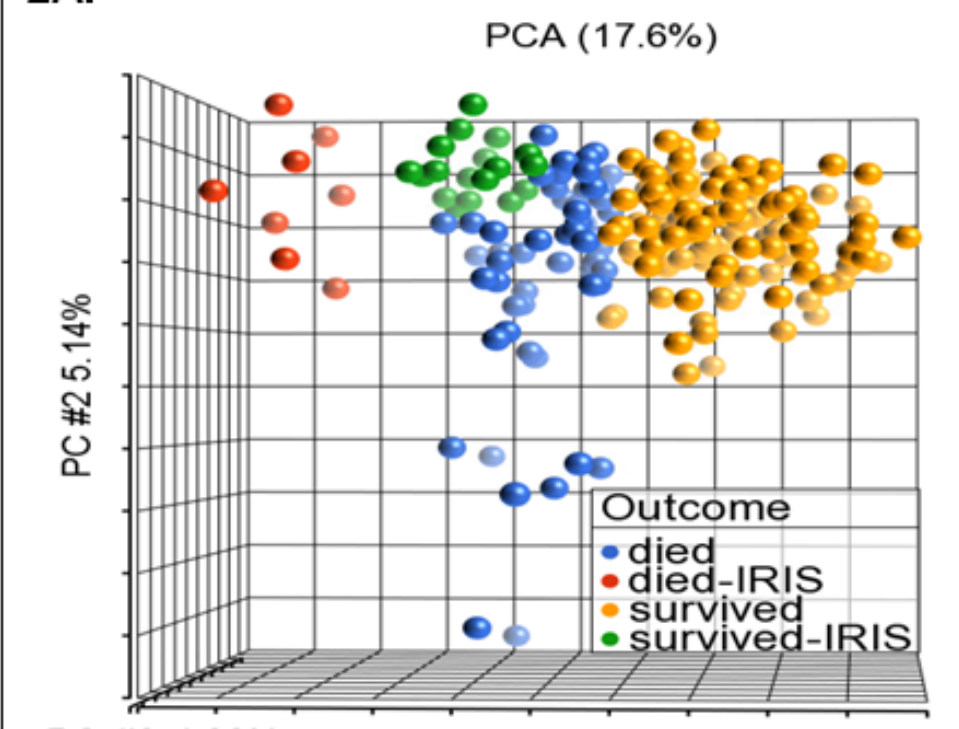

PC \#3 4.66\%

PC \#1 7.77\%
2B.

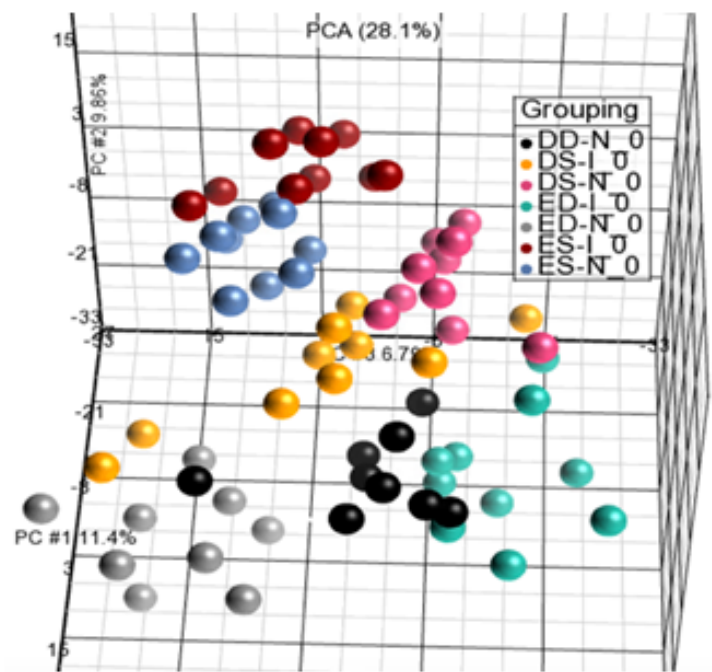

Figure 2

Gene expression patterns distinguish Death, Survival, and C-IRIS. . 2A. Principal component analysis was used to separate gene expression in 211 individual samples from the 68 subjects. 3-D principal components (PC) accounted for $17.6 \%$ total variance in gene expression (PC\#1 7.77\%, PC\#2 5.14\%, PC\#3 $4.66 \%$, respectively). This PCA was performed on 26380 expressed RNAs. The box within the plot outlines the color assignments. Samples, collected the time of C-IRIS events are colored as red (died-IRIS = Fatal C-IRIS Group) and green (survived-IRIS = C-IRIS Survivor Group). Samples shown in yellow are from patients who survived (excluding samples collected at the time of C-IRIS events. Samples shown in blue are from those who died (excluding samples collected at the time of C-IRIS events. For the simplicity of representation, the longitudinally assessed time points within each group are not colored differently. 2B. PCA subgroup analysis at baseline (at randomization, week 0). This PCA was performed on 2200 expressed RNAs that encode proteins with immune functions. The legend in the plot outlines the color assignment: samples were color-coded by the 7 study groups as shown in the box within the figure: DD-N = Deferred Arm, Death without C-IRIS Group (black); DS-I = Deferred Arm, C-IRIS Survivor Group (yellow); DS-N = Deferred Arm, No C-IRIS or Death Group (pink); ED-I = Earlier Arm, Fatal C-IRIS Group (green); ED-N = Earlier Arm, Death without C-IRIS Group (grey); ES-I = Earlier Arm C-IRIS Survivor Group (maroon); and ES-N = Earlier arm, No Death or C-IRIS Group (blue). 


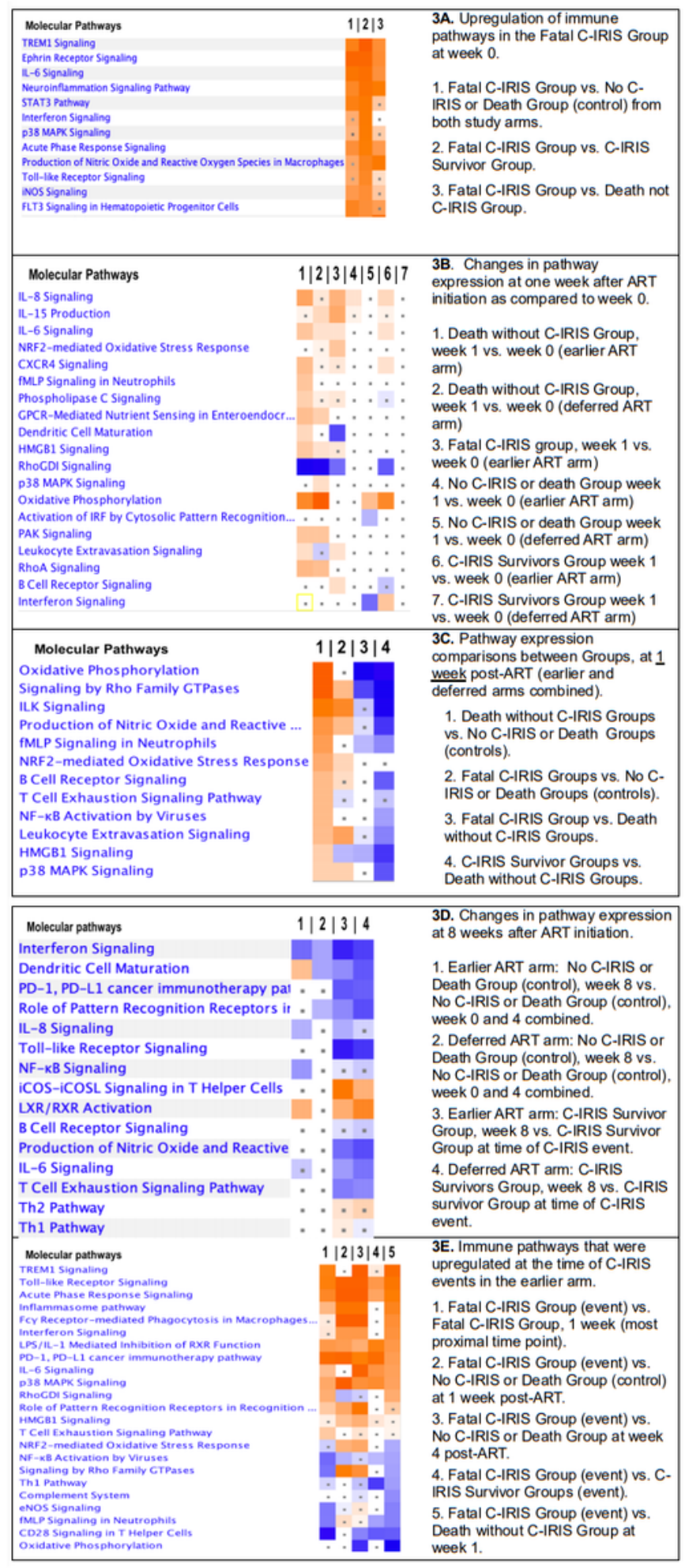

\section{Figure 3}

Comparisons of the expression of immune genes and immune/inflammatory pathways between groups. Biofunction heatmap illustration of pathway-level enrichment based on ANOVA analysis. IPA software visualized the comparison of enriched canonical pathways. Heatmaps display top up- and downregulated pathways. The color of heatmap squares represents the activity of biofunctions. The z-scores represent the significance of fold change expressions and colored as activation z-score $>2$ (orange 
squares), repression z-score <-2 (blue), and insignificant z-score (white squares). Dots mark the squares which did not pass Benjamini-Hochberg restrictions for the pathway that involved in gene expression changes. Vs., versus. Ranges of z-scores identified in this analysis:

\section{Transcriptomic biomarker pathways of Fatal C-IRIS}

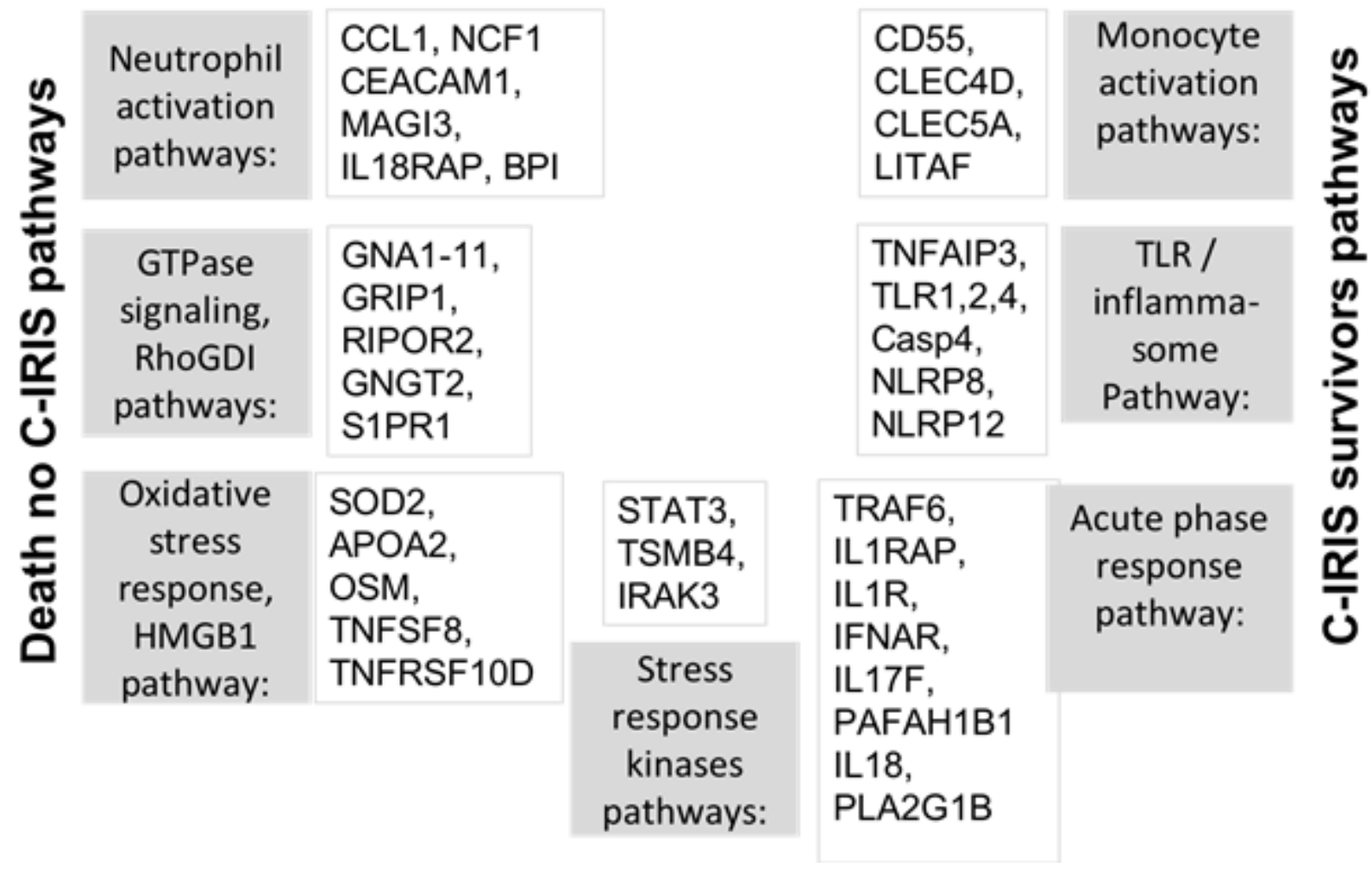

\section{Figure 4}

Transcriptional biomarkers and biomarker pathways that drive fatal inflammation during C-IRIS events. These biomarker transcripts were identified using a partial least square algorithm. The pathways that play a role in fatal outcome are depicted in grey boxes. The transcripts that are unique to the Fatal C-IRIS Group are depicted in clear boxes. The pathways, shared with the Death without C-IRIS Group are depicted on the left; the pathways shared with the C-IRIS Survivor Group are depicted on the right. Pathway, shared by all three groups is depicted on the bottom. Our model suggests that the overactivated innate immune system, driven by monocytes via TLR/inflammasome pathways, drives C-IRIS events (right). Excessive neutrophil activation via fMLP, Rho family GTPases, HMGB1 and inactive RhoGDI pathways results in cellular and tissue damage promoting the pathogenesis of fatal events (left).

\section{Supplementary Files}

This is a list of supplementary files associated with this preprint. Click to download.

- SupplementalTable1ANOVA.xlsx

- SupplementalTable2DiedNoIRISweekOBMCG.xIsx 
- SupplementalTable3PLSbiomarkers.xIsx

- SupplementalFigure1.pptx

- SupplementalFigure2.docx 